\title{
SynergyFinder Plus: towards a better interpretation and annotation of drug combination screening datasets
}

Shuyu Zheng ${ }^{1}$, Wenyu Wang ${ }^{1}$, Jehad Aldahdooh ${ }^{1}$, Alina Malyutina ${ }^{1}$, Tolou Shadbahr ${ }^{1}$, Alberto Pessia $^{1}$ and Jing Tang ${ }^{*}$

${ }^{1}$ Research Program in Systems Oncology, Faculty of Medicine, University of Helsinki

* Correspondence email address: jing.tang@helsinki.fi

\section{Abstract}

Combinatorial therapies have recently been proposed for improving anticancer treatment efficacy. The SynergyFinder R package is a software tool to analyse pre-clinical drug combination datasets. We report the major updates to the $\mathrm{R}$ package to improve the interpretation and annotation of drug combination screening results. Compared to the existing implementations, the novelty of the updated SynergyFinder R package consists of 1) extending to higher-order drug combination data analysis and the implementation of dimension reduction techniques for visualizing the synergy landscape for an unlimited number of drugs in a combination; 2) statistical analysis of drug combination synergy and sensitivity with confidence intervals and p-values; 3 ) incorporating a synergy barometer to harmonize multiple synergy scoring methods to provide a consensus metric of synergy; and 4) incorporating the evaluation of drug combination synergy and sensitivity simultaneously to provide an unbiased interpretation of the clinical potential. Furthermore, we enabled fast annotation for drugs and cell lines that are tested in an experiment, including their chemical information, targets and signalling network information. These annotations shall improve the interpretation of the mechanisms of action of drug combinations. To facilitate the use of the $\mathrm{R}$ package within the drug discovery community, we also provide a web server at www.synergyfinderplus.org that provides a user-friendly interface to enable a more flexible and versatile analysis of drug combination data.

\section{Introduction}

Many complex diseases, including cancers and infectious diseases, usually develop drug resistance easily ${ }^{1,2}$. To achieve more sustainable clinical efficacy, multi-targeted drug combinations are proposed to tackle the disease signalling pathways more systematically ${ }^{3}$. With the advances of high-throughput drug screening technologies, an increasing number of drug combinations is being 
tested in multiple disease models, such as cancer cell lines and patient-derived primary cultures. The main aim of these preclinical drug combination experiments is to identify the most synergistic and effective drug combinations that result in improved cellular responses, such as cell viability and toxicity. With the potential hits prioritized from the screening, more functional studies can be warranted to elucidate the mechanisms of action of the drug interactions that ultimately lead to the identification of drug combination response biomarkers that are critical for stratifying patients for more targeted therapies.

To evaluate the potential of a drug combination, mathematical and statistical models are needed to characterize the expectation if the drugs are not interactive, after which the boosting effects of the drug combination can be formally quantified. There are currently four major synergy models: the highest single agent (HSA) ${ }^{4}$, LOEWE $^{5}$, BLISS ${ }^{6}$ and ZIP ${ }^{7}$. However, the overall effects of a drug combination may not lead to sufficient efficacy despite a strong degree of interaction. Therefore, it is recommended to evaluate both synergy (i.e. the degree of interactions) and sensitivity (i.e. the overall treatment efficacy) simultaneously ${ }^{8}$. Previously developed tools that can analyse drug combination synergy include COMPUSYSN ${ }^{9}$, BRAID $^{10}$, Combenfit ${ }^{11}$, SynergyFinder ${ }^{12}$ and Synergy ${ }^{13}$. However, these tools are designed mainly for analysing two-drug combinations only. More recently, the SynergyFinder 2 tool ${ }^{14}$ has been developed, which extends to the three-drug combinations. However, only the HSA model is compatible with its lower-order scenarios in its implementation. Furthermore, the visualization of the high-order drug combinations becomes nontrivial, as none of the existing visualization methods can deal with combinations of three or more drugs ${ }^{15}$. More importantly, there is a lack of implementation tools that can harmonize the multiple synergy models to derive a consensus about the degree of interactions ${ }^{16}$.

To address these limitations, we provide a major update to the SynergyFinder R package that enables novel functions: 1) formal mathematical models to assess the synergy of high-order drug combinations; 2) visualization of the degree of synergy using a dimension reduction technique; 3) formal statistical methods to evaluate the significance of synergy; 4) an implementation of the synergy barometer to systematically compare the results from different synergy models; and 5) an implementation of a synergy-sensitivity (SS) plot to evaluate the potential of a drug combination unbiasedly. Finally, we developed data annotation tools to retrieve the pharmacological and molecular profiles of the drugs and cells, which shall facilitate the discovery of mechanisms of action of the most synergistic and effective drug combinations. We provide a new website at www.synergyfinderplus.org to allow a user to upload their experimental results and run all the 
analyses with the R package in the backend. All the functions and source codes are freely accessible for academic users (Figure 1).

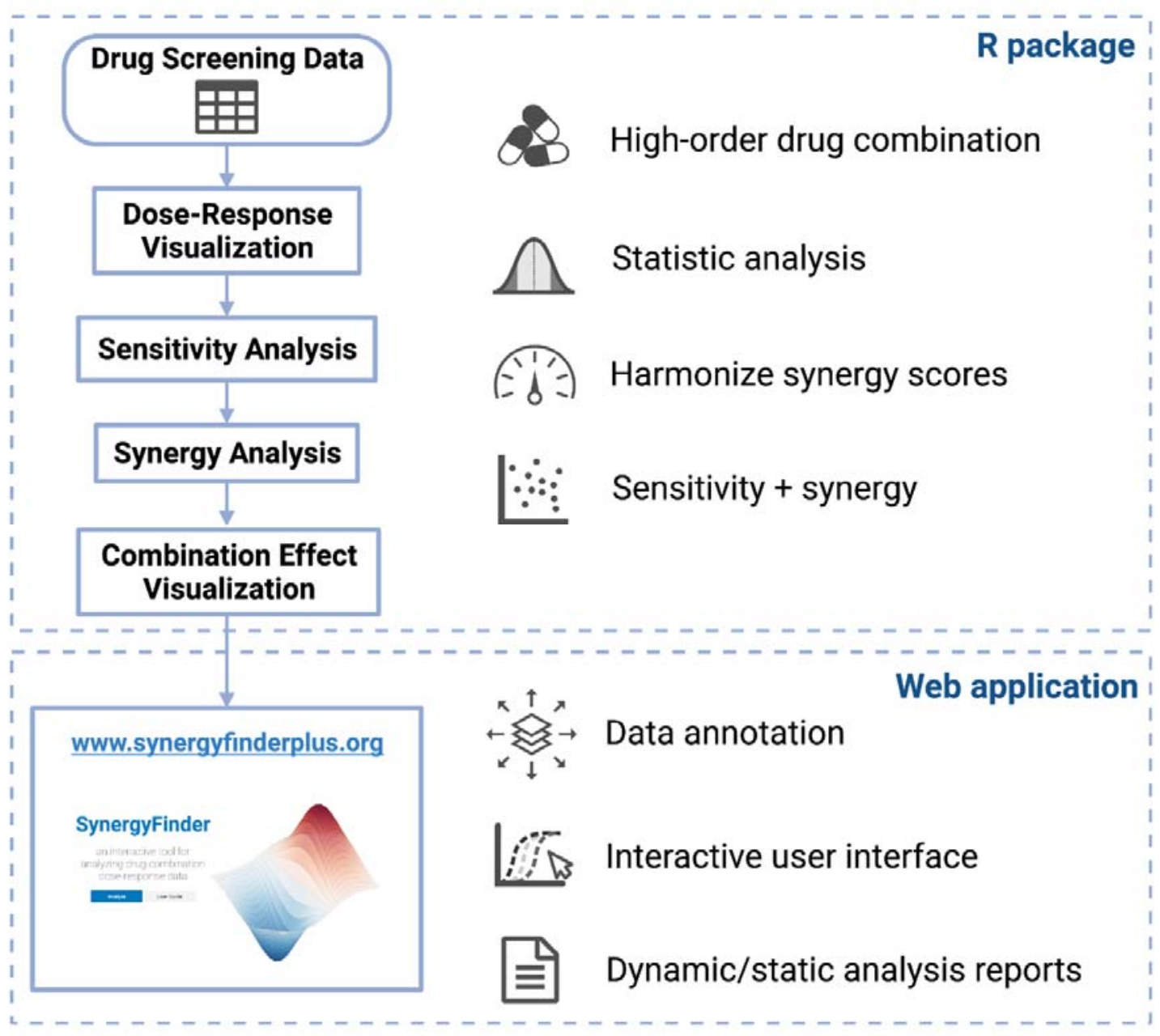

Figure 1. Summary of the SynergyFinder Plus workflow. We highlight the new features: 1) the capability of analysing drug combinations of more than two drugs; 2) tailored statistical testing to account for uncertainty for data with replicates; 3 ) harmonized visualization of multiple synergy score metrics; 4) visualization of the relationship between sensitivity and synergy scores; and 5) a data annotation web server to query multiple cell line and drug databases.

\section{Methods}

\subsection{Synergy and sensitivity models for high-order drug combinations}

Consider the response of a drug to be measured as a $\%$ inhibition that ranges from 0 to 1 , with a higher value indicating better efficacy. For a combination that involves drugs, the observed combination response is denoted as , while the observed monotherapy response of its constituent 
drugs is $y_{i, i=1, \ldots n}$. The expected combination response is determined by the assumption of noninteraction. Currently there are four major synergy reference models ${ }^{16,17}$. For the HSA model, the expected response is the highest monotherapy response, i.e. $y_{H S A}=\max \left(y_{1}, \ldots, y_{i}, \ldots, y_{n}\right)$. For the BLISS model, the expected response is derived from the probabilistic independence of the monotherapy effects, i.e. $y_{B L I S S}=1-\prod_{i}\left(1-y_{i}\right)$. For the LOEWE model, the expected response satisfies $\sum_{i}\left(\frac{x_{i}}{f_{i}^{-1}\left(y_{\text {LOEWE }}\right)}\right)=1$, where $x_{i}$ is the dose of each constituent drug in the combination and $f_{i}^{-1}\left(y_{\text {LOEWE }}\right)$ is the inverse function of the dose response curve. For the ZIP model, the expected response satisfies $y_{Z I P}=1-\prod_{i}\left(1-\hat{y}_{i}\right)$, where $\hat{y}_{i}$ is the predicted dose response of the monotherapy by a monotonically increasing curve fitting model $\hat{y}_{i}=f_{i}\left(x_{i}\right)$. For example, a common choice for modelling drug dose response curves is the four parameter log logistic model $f(x)=\frac{E_{\min }+E_{\max }(x / m)^{\lambda}}{1+(x / m)^{\lambda}}$, where $E_{\min }, E_{\max }, m$ and $\lambda$ are the minimal and maximal responses, IC50 and slope of the dose response curve, respectively.

Accordingly, the multi-drug synergy score for the observed combination response $y_{c}$ can be determined as:

$$
\begin{gathered}
S_{H S A}=y_{c}-\max \left(y_{1}, \ldots, y_{i}, \ldots, y_{n}\right) . \\
S_{\text {BLISS }}=y_{c}-\left(1-\prod_{i}\left(1-y_{i}\right)\right)=y_{c}-\left(\sum_{i} y_{i}-\sum_{i<j} y_{i} y_{j}+(-1)^{r+1} \times\right. \\
\left.\sum_{i<j} \prod_{j}^{r} y_{j}+(-1)^{n+1} \times \prod_{i} y_{i}\right) \\
S_{\text {LOEWE }}=y_{c}-y_{\text {LOEWE }}, \text { s.t. } \sum_{i}\left(\frac{x_{i}}{f_{i}^{-1}\left(y_{\text {LOEWE }}\right)}\right)=1
\end{gathered}
$$

For determining the ZIP-based synergy score, $y_{c}$ needs to be replaced with the predicted average response $\hat{y}_{c}$ given by the curve fitting models $f_{i}^{\prime}$ to make it comparable to $y_{Z I P}$ :

$$
S_{Z I P}=\widehat{y}_{c}-y_{Z I P}=\frac{1}{n} \sum_{i} f_{i}^{\prime}\left(x_{i}\right)-\left(1-\prod_{i}\left(1-f_{i}\left(x_{i}\right)\right),\right.
$$

$$
\text { where } \operatorname{Emin}\left(f_{i}^{\prime}\right)=f_{-i}^{\prime}\left(x_{-i}\right)
$$

Namely, $f_{i}^{\prime}(x)$ stands for the log logistic model defined for the combination response at dose $x$ of drug $i$ when the other drugs are present. Furthermore, Emin of $f_{i}^{\prime}(x)$ is determined by $f_{-i}^{\prime}\left(x_{-i}\right)$, which is the fitted curve of the combination response while drug $i$ is absent. Note that the ZIP model captures the shift of potency for a drug combination in comparison to its monotherapy drugs, therefore, the ZIP model compares the difference of fitted models for the drug combination $f_{i}^{\prime}\left(x_{i}\right)$ and for the monotherapy drugs $f_{i}\left(x_{i}\right)$. 


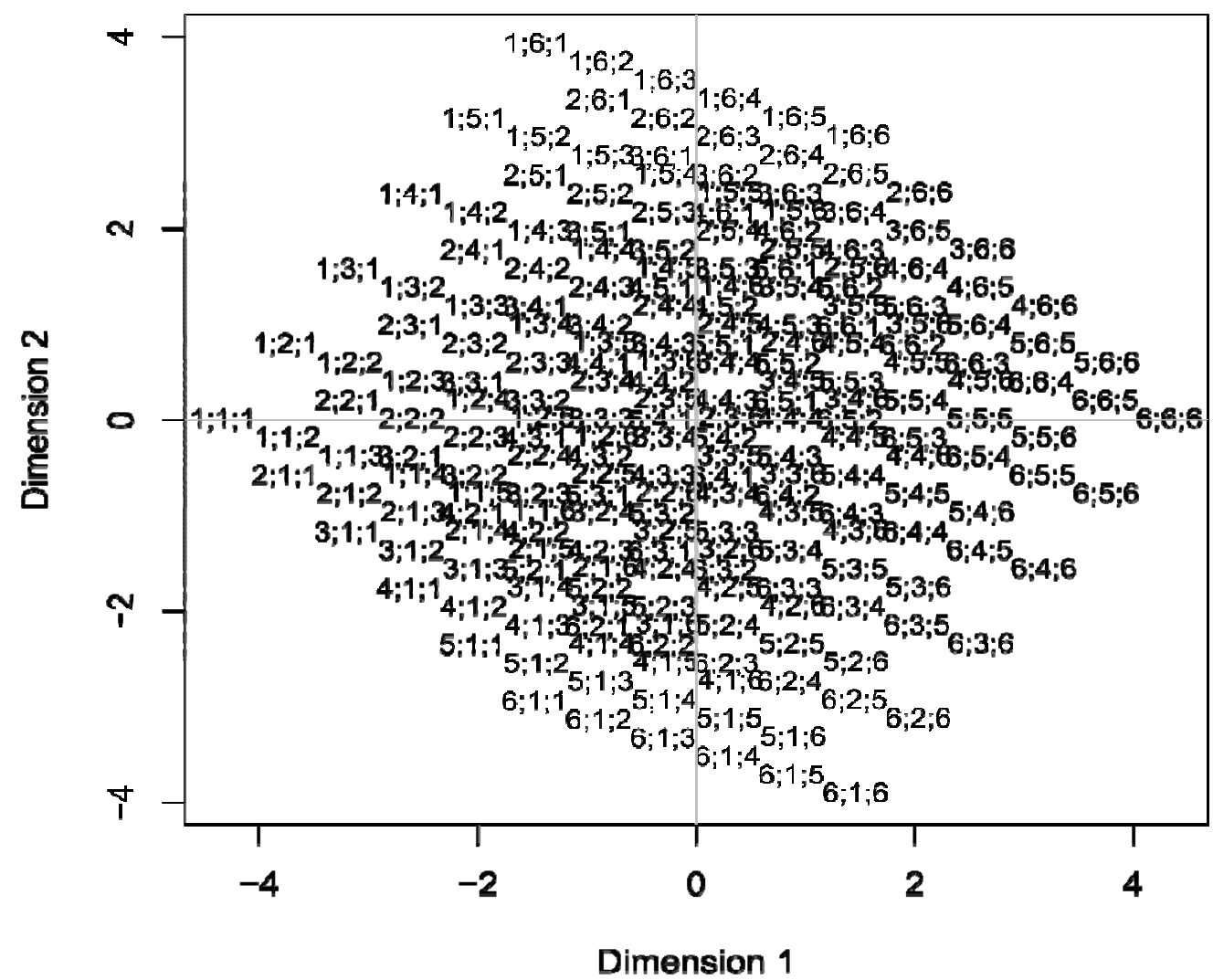

Figure 2. Dimension reduction for the visualization of high-order drug combinations. In this example, the two-dimensional coordinates of each three-drug dose combination are determined by multi-dimensional scaling based on the similarity of their dose ranges, after which the synergy scores and sensitivity scores can be visualized on the map. Each dot in the map is annotated with its associated dose rankings for the three drugs . For example, a dot with a label 4;1;2 shows a combination involves 4 th dose of drug $1,1^{\text {st }}$ dose of drug 2 and $2^{\text {nd }}$ dose of drug 3 .

\subsection{Visualization of higher-order synergy and sensitivity scores}

For the visualization of higher-order drug combinations on a map of dose configurations, the commonly utilized synergy or sensitivity landscape models are not directly applicable. Here we propose a dimension reduction technique that is based on multi-dimensional scaling, similar to the recent application in transforming numerical data into images ${ }^{18}$. For a drug combination in a highdimensional dose space, with its coordinates of , we utilize their rankings

to determine the pairwise similarity between the instances of . A common choice of quantifying the similarity is the Euclidean distance. We utilize multi-dimensional scaling to minimize the error of the pairwise distance in a two-dimensional space in which the synergy and sensitivity scores can be visualized as a synergy landscape (Figure 2). Note that using the dose rankings as the input for multi-dimensional scaling can assure that the resulting two-dimensional 
coordinates are equally distanced among the neighbouring dose conditions, thus making the visualization easier to interpret. Furthermore, for the case of two-drug combinations, the algorithm can converge to the actual dose rankings, thus preserving the consistency across all the orders of combinations. We have also developed functions to visualize the synergy and sensitivity scores for a specific dose condition in a grid of barplots, which can help identify the most synergistic and sensitive areas in the synergy landscape.

\subsection{Statistical analysis}

The statistical significance of synergy can be defined at a single dose or at the whole dose matrix level. Take a two-drug combination experiment as an example. We assume that the replicates of drug responses are measured independently within each dose in the dose matrix (Figure 3). At each dose level, we propose using a bootstrapping approach to determine the confidence intervals of the synergy scores. Namely, we sample with replacement from the replicates to determine a bootstrap dose-response matrix. The synergy scores for the HSA and BLISS models can be derived simply by comparing the bootstrapped responses at the dose combination and at their corresponding monotherapy doses (i.e. using eq (1) and eq (2)). However, for the LOEWE and ZIP models, bootstrapped synergy scores will be derived using the curve fitting over the whole dose matrix (i.e. eq (3) and eq (4)).

Suppose that $B$ bootstrap samples are drawn from the replicates, and suppose that $s_{1}, \ldots, s_{B}$ are the estimates of the synergy scores determined from these bootstrap samples, with mean of $\bar{s}$. We determine the bootstrap standard error as

$$
S E=\sqrt{\frac{1}{B-1} \sum_{i=1}^{B}\left(s_{i}-\bar{s}\right)^{2}}
$$

The $95 \%$ confidence interval for the synergy score is approximately

$$
[\bar{s}-1.96 S E, \bar{s}+1.96 S E]
$$

Meanwhile, at the whole dose matrix level, we provide an empirical p-value to assess the significance of the difference between the estimated average synergy score over the whole dose matrix and the expected synergy score of zero under the null hypothesis of non-interaction.

Letting $s^{\prime}{ }_{1}, \ldots, s_{B}^{\prime}$ be the estimates of the average synergy scores over the whole dose matrix from the bootstrap samples, the $\mathrm{p}$-value is

$$
p=\exp \left(-0.717 z-0.416 z^{2}\right), \text { where } z=a b s\left(\bar{s}^{\prime}\right) / \sqrt{\frac{1}{B-1} \sum_{i=1}^{B}\left(s_{i}{ }^{\prime}-\bar{s}^{\prime}\right)^{2}}{ }^{19} .
$$


For a scenario where no replicates are available, the confidence interval cannot be determined at the dose level. However, the p-value of the average synergy score of the whole dose-response matrix can still be derived by pooling the synergy scores together and then comparing them to zero. For both scenarios, we report the overall p-values of synergy and the confidence intervals at the dosespecific level when replicates are available.

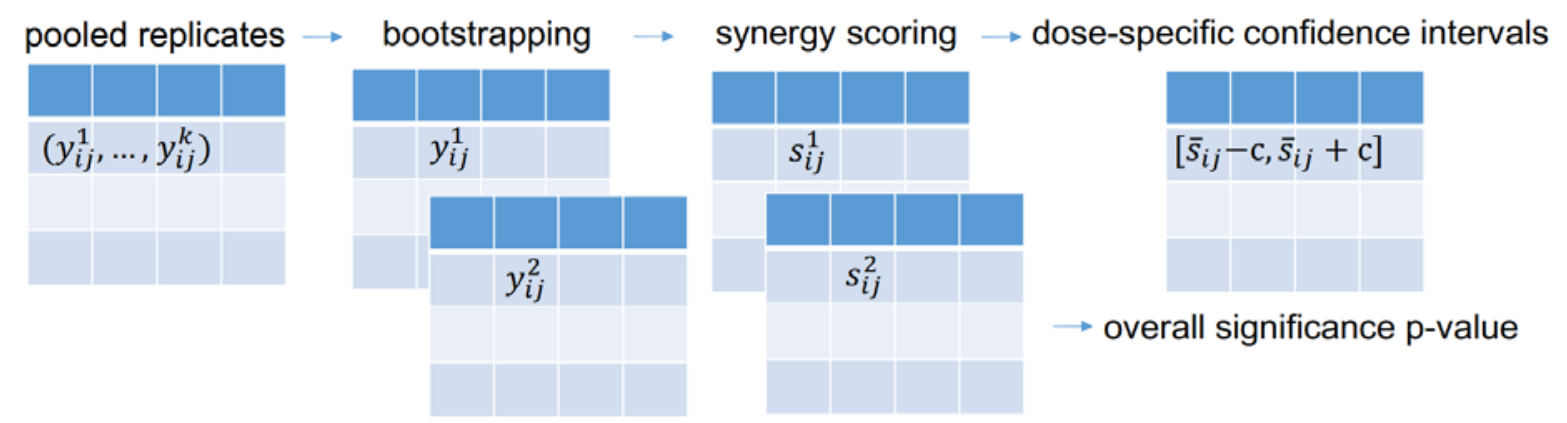

Figure 3. Statistical testing of synergy scores. Dose-specific confidence intervals as well as p-value for the average synergy score can be derived using bootstrapping of the dose-response matrix.

\subsection{Harmonization of multiple synergy models}

As the SynergyFinder R package provides four synergy models (i.e. HSA, BLISS, LOEWE and ZIP) that differ in their null hypotheses of non-interaction, the synergy scores do not necessarily coincide with each other for a given dataset. In order to provide a more harmonized representation of the synergy score results, we introduce here a novel tool to enable a more systematic comparison of the synergy scores. We leveraged the implementation of the four models so that the expected response of non-interaction (i.e. $y_{H S A}, y_{B L I S S}, y_{L O E W E}$ and $y_{Z I P}$ ) could be derived. Since all the expected responses share the same unit as the observed response (i.e. \% inhibition), we may utilize a synergy barometer to compare these values. The expected and observed responses for a given combination at a specific dose condition are positioned on the same scale. With such a tool as the synergy barometer, one cannot only evaluate the degree of synergy of a specific model, but also easily understand the differences in the results among them. Ideally, a strong synergy should be concluded if the observed response goes beyond the expected responses of all four models.

\subsection{Prioritization of drug combinations}

In addition to synergy, the sensitivity of a drug combination is equally important ${ }^{8}$. Prioritization of drug combinations based on the degree of synergy may only result in an excessive number of false positives, as a clinical endpoint for approving a drug combination is usually its efficacy rather than the synergy. In cases where a strong synergy is obtained, the drug combination may not necessarily 
reach sufficient levels of therapeutic efficacy. To capture the sensitivity of a drug combination, we have previously developed a combination sensitivity score (CSS) model that calculates the relative inhibition of a drug combination based on the area under the $\log 10$ scaled dose response curves at the IC50 doses of the constituent drugs ${ }^{8}$. The CSS has the same unit as a drug response (i.e. $\%$ inhibition), which makes it directly comparable with the synergy scores that are defined in equations (1-4). Therefore, we propose a scatter plot of the CSS versus synergy score for a set of drug combinations. The so-called sensitivity-synergy (SS) plot shall greatly aid in the prioritization of drug combinations from a high-throughput experiment.

\subsection{Annotation of drug combinations}

Drugs and cell lines in a drug screening dataset are annotated by querying the related databases. Drug identifiers are extracted from multiple major chemical compound databases, including PubChem CID, ChEMBL ID, InChIKey and SMILES. DrugBank ID and KEGG ID are further extracted from UniChem ${ }^{20}$. The clinical stage for a given drug is extracted from both ChEMBL and UniChem. In cases of inconsistencies between the two databases, the later clinical stage is used. Furthermore, we provide drug target profiles from DrugTargetCommons ${ }^{21}$. The cell line annotation is obtained from Cellosaurus ${ }^{22}$ (Version 37.0).

\section{Results}

\subsection{Analysis of three-drug combinations}

To test the algorithms designed for higher-order combinations, we utilized one of the recent antimalaria studies in which 16 triple artemisinin-based combination therapies have been tested against 15 parasite lines ${ }^{23}$. The aim of this study was to identify synergistic partner drugs that can be combined with artemisinin to overcome the emergence of malaria resistance. For each triple drug combination, a 10x10x12 multi-dimensional dose matrix was constructed, resulting in $\mathrm{n}=1200$ viability values. The datasets were obtained from the NCATS data portal at https://tripod.nih.gov/matrix-client/.

We analysed the triple drug combinations in terms of their sensitivity and synergy and visualized them in surface plots using the dimension reduction techniques. Figure 4 shows a synergistic triple combination, Pyronaridine Tetraphosphate-Artesunate-Darunavir Ethanolate (PYR-ATM-DRV) compared to an antagonistic combination, Pyronaridine Tetraphosphate-Piperaquine-Lopinavir 
(PYR-PQP-LPV). It can be seen that despite the similar sensitivities (the average \% inhibition is 78.87 for PYR-ATM-DRV and 74.97 for PYR-PQP-LPV), their synergy scores differ drastically (the average ZIP score is 11.72 for PYR-ATM-AQ and -13.05 for PYR-PQP-LPV), suggesting more complex behaviours of the drug interactions as the number of drugs increases. Note that due to the lack of computational tools for analysing higher-order drug combinations, the authors of the anti-malaria studies provided only the results of pairwise drug combinations, whereas the actual interactions of the three drugs were left unexplored. Our method therefore may provide novel insights that leverage the higher-order drug combination design in a more tailored manner.

A

Dose Response Matrix
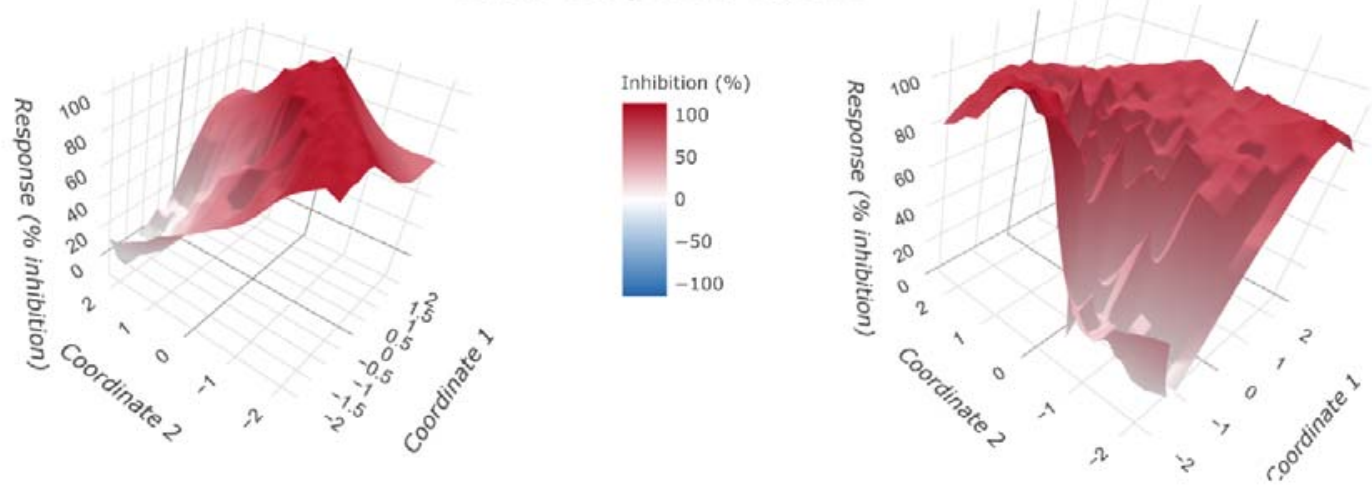

B

ZIP Synergy Score
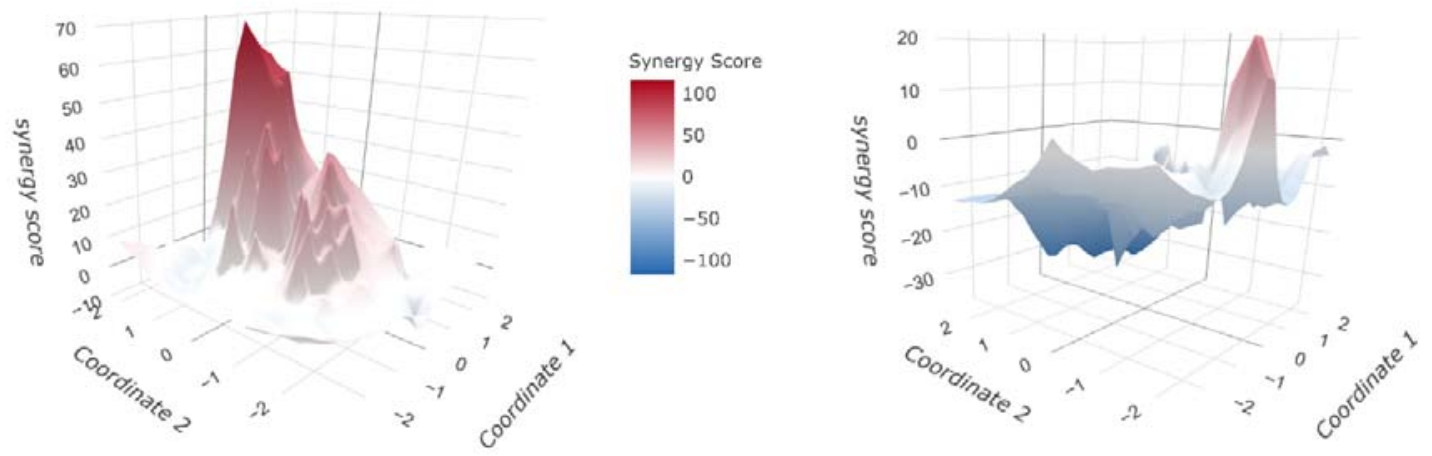

Figure 4. Example of surface plots for higher-order drug combinations. A) The dose response map and B) the ZIP synergy score map for a synergistic drug combination (Pyronaridine Tetraphosphate-Artesunate-Darunavir Ethanolate, left panel) compared to an antagonistic drug combination (Pyronaridine Tetraphosphate-Piperaquine-Saquinavir, right panel). For surface plots of other synergy scores, including HSA, BLISS and LOEWE, see Supplementary Figure 1. 
We obtained the ONEIL dataset from the DrugComb data portal ${ }^{24}$. The ONEIL data is a pan-cancer drug combination study in which 583 drug combinations have been tested across 39 cell lines ${ }^{25}$. For each drug combination, four replicates have been produced, making it a representative example dataset for the analysis of the significance of the synergy scores. We show selective drug combinations that were tested in the A2058 cell line (melanoma).

As shown in Figure 5A, the confidence intervals for the responses as well as synergy scores vary at different doses, suggesting that not all the scores are equally significant. Furthermore, we observed a general trend that a higher response or synergy score tends to have a smaller confidence interval, consistent with the expectation of a typical drug screening experiment. In order to evaluate the synergy score more systematically, we provided the synergy barometer for specific dose combinations. For example, for MK-1775 at $0.2 \mathrm{uM}$ combined with Niraparib at $2.75 \mathrm{uM}$, the drug combination response reaches $74.84 \%$ inhibition, as indicated by the pointer readout on the barometer (Figure 5B, left panel). Such a drug combination response is synergistic, as the expected responses of the HSA, LOEWE, BLISS and ZIP models are much smaller, shown as the marks on the barometer. In contrast, Paclitaxel at $0.0016 \mathrm{uM}$ in combination with L-778123 at $2 \mathrm{uM}$ shows a near zero response ( $0.29 \%$ inhibition), which is much smaller than the expected response among all the synergy models (Figure 5B, right panel); therefore, it is considered strong antagonism. With the tool of the synergy barometer, the actual drug combination response can be straightforwardly compared with the expectations of non-interaction among multiple synergy models.

Furthermore, we also show the CSS and ZIP synergy score of all the drug combinations that were tested in the A2058 cell line in an SS plot (Figure 5C, Supplementary Figure 2). The CSS indicates the efficacy of a drug combination, whereas the synergy score indicates the degree of interactions. To prioritize potential drug combinations, it is necessary to identify those with both higher CSS and higher synergy scores, i.e. the top-right corner of the SS plot. 
A

Inhibition $(\%)$

$\begin{array}{llll}75 & 50 & 25 & 0\end{array}$

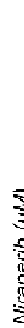

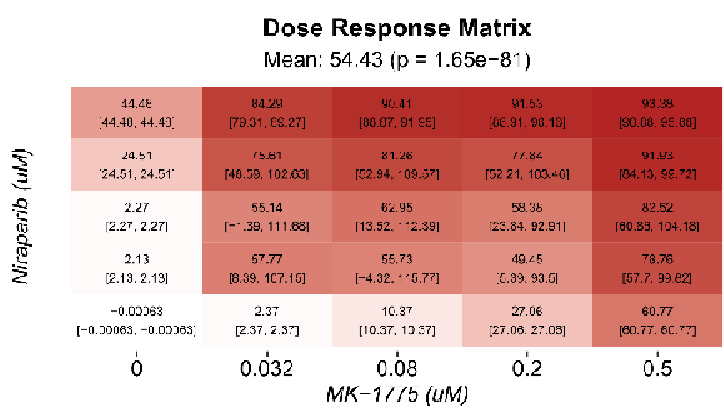

Dose Response Matrix

Mean: $16.8(p=4.16 e-76)$

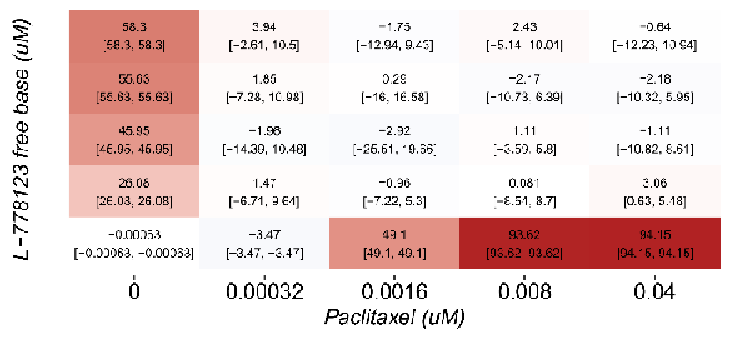

B

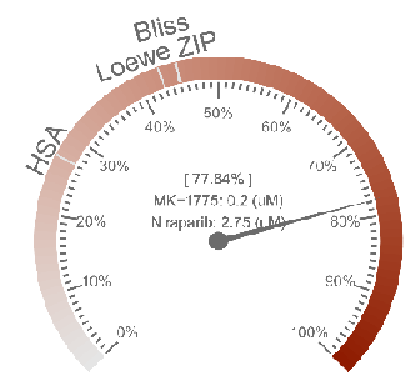

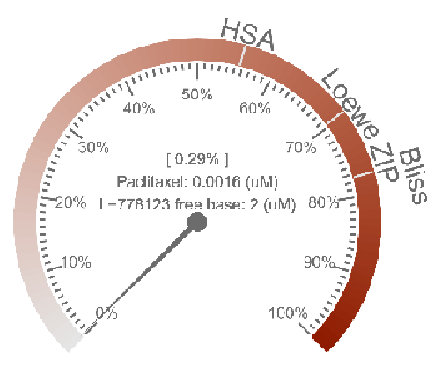

$\begin{array}{llllllll}40 & 20 & 0 & -20 & -40 & -60 & -80 & -100\end{array}$ Synergy Score
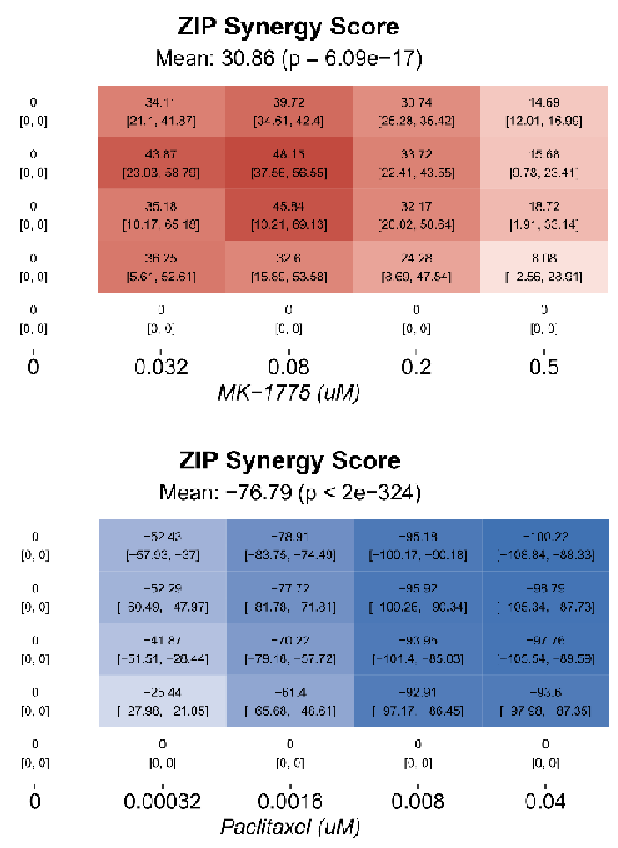

C

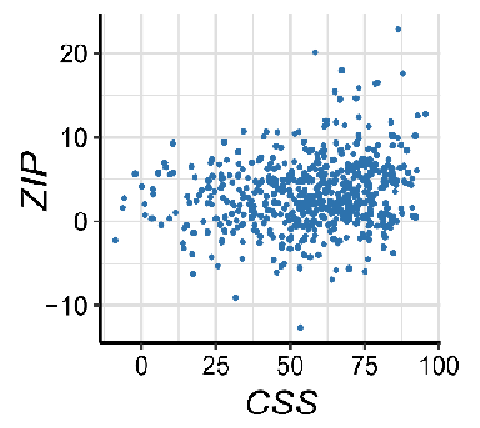

Figure 5. A) An example of the confidence interval plots of a synergistic drug pair (MK-1775 and

Niraparib; upper panel) and an antagonistic drug pair (Paclitaxel and L-778123; lower panel). B)

The synergy barometer for a given dose combination (left panel: MK-1775 at $0.2 \mathrm{uM}$ in combination with Niraparib at $2.75 \mathrm{uM}$; right panel: Paclitaxel at $0.0016 \mathrm{uM}$ in combination with L778123 at $2 \mathrm{uM})$. C) The synergy-sensitivity plot for drug pairs tested in the A2058 cell line.

\subsection{Annotation of mechanisms of action of drug combinations}

Recent advances in machine learning and artificial intelligence studies have shown great potential in terms of predictive modelling of drug synergies ${ }^{26-28}$. Naturally, in these studies, the chemical structure information about drugs and molecular profiles of cells are considered as predictive features. However, for most of the drug combination datasets, the annotations of drug and cell 
features are not provided. To facilitate the interpretation of mechanisms of action of drug combinations, it would therefore be beneficial to provide data integration tools for effective annotation and accurate matching to public databases such that more comprehensive features used for synergy predictions can be obtained. Efforts regarding annotating and harmonizing existing drug screening data, such as DrugComb ${ }^{24,29}$ and DrugCombDB ${ }^{30}$, have significantly contributed to the development of data-driven pharmacological modelling ${ }^{31-33}$. For newly generated drug screening datasets, we have developed the SynergyFinder Plus portal further as a companion tool for retrieving publicly available information in a more automated fashion. With one click of the button, users can obtain not only cross-database identifiers of the drugs and cells, but also additional information, such as the mechanism of action and disease indication.

Here we show how SynergyFinder Plus annotates a particular drug combination in the ONEIL ${ }^{25}$ dataset (5-Fluorouracil and Vorinostat tested in the A2058 cell line, Figure 6A). After users upload the dataset, SynergyFinder Plus returns two annotation tables describing the cell and drug identities. For example, the cell annotation table includes synonyms, Cellosaurus accession, tissue origin, disease name and disease NCI Thesaurus ID, whereas the drug annotation table includes PubChem CID, ChEMBL ID, InChIKey, SMILES, DrugBank ID, KEGG ID and the clinical trial phase. Additionally, a third table listing detailed drug-target binding information is provided, allowing the biological processes of drug perturbation to be further retrieved using the target identifiers in ChEMBL. These annotations enable a more systematic exploration of drugs and cell lines that are tested in a high-throughput experiment (Figure 6B). With such a comprehensive collection of identifiers, we believe that the drug combination data can be better integrated with other related data sources for further downstream analysis. 
A

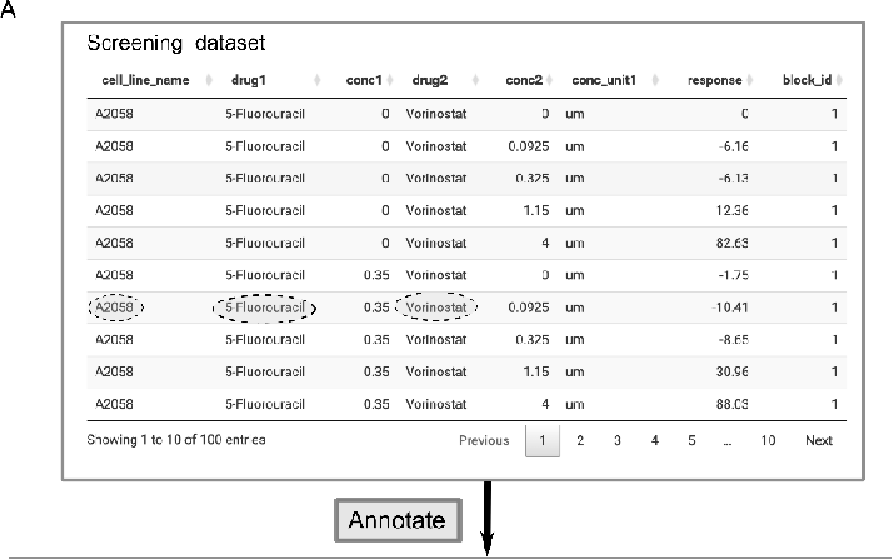

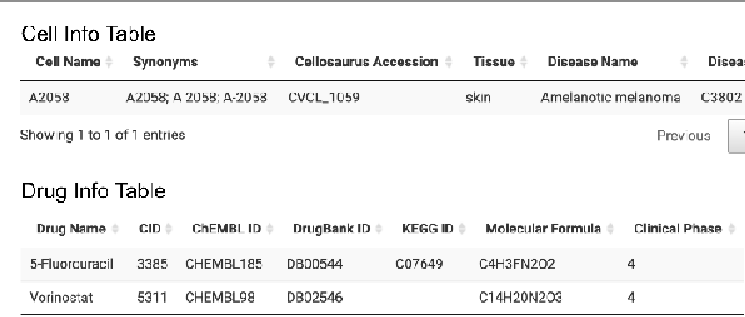

Showing 1 to 2 of 2 on:rices

MoA Info Tasle

\begin{tabular}{|c|c|c|c|c|c|}
\hline Drug Name & ChEMEL ID & Target Gene Name & Target Protain Name & Targget Protoin Class & Targot Drganism \\
\hline 5-luarruliccil & G-EMBL' 85 & GEKAR & CHOLECYSTOKININ A REGEPTOF & GFCR & Ho no sapiaris \\
\hline $5=$ =luarourzecll & CARMBL 8.5 & $\mathrm{HFH}_{2}$ & HISTAMINE HZ RECEPTOR & GFCR & H.วno saplens \\
\hline Vorinostat & CAEMBL 98 & $H D A C^{-}$ & HISTONE DEACETYLSSE 1 & Epigenetic regulastor & Hono sapiens \\
\hline Vorinostat & CHEMBL28 & HDACG & HISTONE DEACETYLASE G & Épigenetic regulettrr & Hamo sapiens \\
\hline Yorinustat & C-AEMBL98 & HDAC3 & HISTONE DEACETYLASE 3 & Epigenelic regulator & Нэто sapiers \\
\hline Yoninnstat & GAFMiRi Q & HILCS & HESTINF GDFACFTVI LSFF? & Enigenetic regullator & Homn sapiens \\
\hline 5 -iverourscil & CABMBL 85 & Trus & THYMIDYLAE ESYNTHASE & Enzzyme & H2 no sapicne \\
\hline 5-7uoroürecil & C IDMBL 75 & & NITR C OXID- SYNTIIAGS, INDUGIBLE & Encyme & Mus muscilus \\
\hline Vorinostat & CHEMBL98 & HMGSR & FMG-COA REDUSTASE & Enzyme & Hวnо saplens \\
\hline vonnostat & C-EMLL98 & & FISIONE DEACE IYLASE & $=n z y m e$ & Rattus norvegıus \\
\hline
\end{tabular}

B
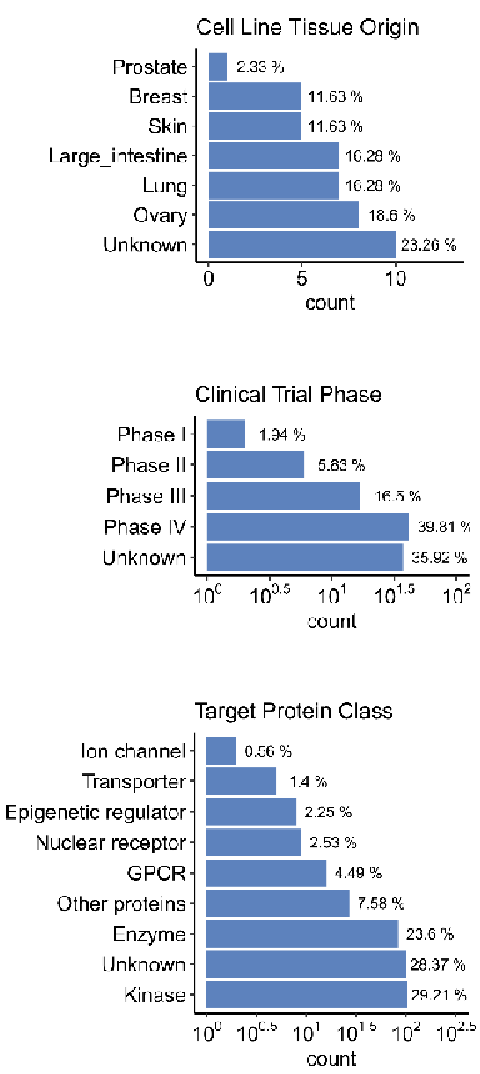

Figure 6. A) An example of a drug screening annotation table. The tested drug combination, 5Fluorouracil and Vorinostat, is further annotated with their identifiers and mechanism of action information. Cell line information for the A2058 is also provided, including synonyms, tissue and disease types. B) Summary of drug clinical stage, drug target protein class and cell line tissue origin for the ONEIL dataset.

\section{Discussion and conclusion}

Synergistic and effective drug combinations have long been sought for improving disease management. Testing of potential drug combinations using clinically representative cell cultures has become an important tool for detecting signs of drug sensitivity and resistance, and in some cases, the results have already provided informative decision-making resources to guide patient stratification in clinical trials ${ }^{34}$. To facilitate the prioritization of drug combinations, informatics tools that enable formal assessment of the degree of synergy as well as sensitivity are highly needed. 
We provide a major update to the commonly used SynergyFinder R package that allows the modelling of drug interactions for any higher-order combinations ${ }^{15}$. Higher-order drug combinations have been recently explored in, for example, lung cancer ${ }^{35}$, colorectal cancer ${ }^{36}$ and ovarian cancer ${ }^{37}$. It is therefore expected that promising multi-drug combinations will enter formal clinical trials for multiple diseases ${ }^{38}$. Furthermore, we have developed statistical analysis of the drug combinations, which is also generally applicable for higher-order combinations. Importantly, the mathematical models for higher-order combinations have been made consistent with those for two-drug combinations, and therefore their scores are readily comparable across different orders. To visualize higher-order combinations, we have developed a dimension reduction technique to display the synergy as well as sensitivity landscape on a two-dimensional plane.

In addition to providing multiple synergy models, we have also addressed the problem of harmonization. With the increasing number of models that have been proposed to characterize drug synergy, there is a more pressing need to help users understand the differences between these models such that a user may make an informed interpretation about the drug combination results. We propose the use of a synergy barometer on which all the synergy models can be systematically compared together with the observed drug combination response. Despite only including the four major synergy models, HSA, BLISS, LOEWE and ZIP, we welcome the incorporation of other synergy models that can readily have their synergy scores put into the barometer to be compared with existing models. With the synergy barometer, we hope that the drug discovery community may derive a consensus on the best reporting guideline for the drug combination data analysis, which has been long sought since the time of the Saariselkä agreement ${ }^{39}$ and thereafter ${ }^{40}$.

We also provide the formal statistical analysis for replicates of drug combinations. The confidence intervals and p-values for all four synergy scores are readily comparable. Even for the cases in which no replicates have been performed, the significance of synergy over all the dose conditions can be provided, thus enabling a statistical evaluation of drug combinations in a primary screen that usually does not provide replicates due to the high number of combinations. Once a drug combination hit passes the initial screening based on the synergy barometer and the statistical significance, we recommend a confirmation screen that involves more doses as well as more replicates so that its synergy can be more formally evaluated. If a user would like to know more about the mechanism of action of the drug combination, we also provide integrative tools to retrieve the chemical structure information as well as other functional annotation of the drugs, which shall help the downstream analysis of the drug combinations. 
Finally, we provide new features to harmonize the assessment of synergy and sensitivity. The synergy models that have been developed in the SynergyFinder R package have the same scale as the drug combination sensitivity method that was developed earlier, thus allowing a direct comparison in an SS plot. We recommend the users consider the SS plot when reporting their drug combination results, as the sensitivity is the clinical endpoint for approving any drug or drug combinations. Importantly, the drug combination sensitivity values also have the same scale as monotherapy drug responses in the unit of percentage inhibition, and therefore we can readily compare a drug combination with a single drug to evaluate their efficacy. With the harmonization of drug combination sensitivity and monotherapy sensitivity, we may provide an integrated data source for developing advanced machine learning approaches for predicting drug sensitivity, such as those drug sensitivity datasets that are deposited in DrugComb ${ }^{24}$. To facilitate the FAIRification (findable, accessible, interoperable and reusable characteristics) of drug combination analysis, we provide a web server at www.synergyfinderplus.org to let users upload the data and obtain the analysis results in the form of reports. Meanwhile, we encourage users to deposit their experimental data in DrugComb at https://drugcomb.org/, currently one of the most comprehensive drug screening databases. With the algorithms and the source code made available through the SynergyFinder R package, we welcome the machine learning community to leverage the harmonized drug combination and monotherapy datasets for more robust and transferable predictions to further facilitate the drug combination discovery ${ }^{27,41-43}$.

\section{Acknowledgements}

The www.synergyfinderplus.org web server is located at the CSC-IT Center for Science in Finland. All the source code for implementing the data analysis and visualization is available as the SynergyFinder R package at https://bioconductor.org/packages/release/bioc/html/synergyfinder.html.

\section{Funding}

This work was supported by the European Research Council (ERC) starting grant DrugComb (Informatics approaches for the rational selection of personalized cancer drug combinations) [No. 716063]; European Commission H2020 EOSC-life (Providing an open collaborative space for digital biology in Europe [No. 824087]; Academy of Finland grant [No. 317680]. The Sigrid Jusélius Foundation grant. WW holds a salaried position funded by University of Helsinki through 
Doctoral Program of Biomedicine (DPBM), and also receives a personal grant from K. Albin Johanssons stiftelse. AM holds a salaried position funded by University of Helsinki through Doctoral Program of Integrative Life Science (ILS).

Conflict of interest statement. None declared.

\section{References}

1. Vasan, N., Baselga, J. \& Hyman, D.M. A view on drug resistance in cancer. Nature 575, 299-309 (2019).

2. Nathan, C. Resisting antimicrobial resistance. Nature reviews. Microbiology 18, 259-260 (2020).

3. Boshuizen, J. \& Peeper, D.S. Rational Cancer Treatment Combinations: An Urgent Clinical Need. Molecular cell 78, 1002-1018 (2020).

4. Berenbaum, M.C. What is synergy? Pharmacological reviews 41, 93-141 (1989).

5. Loewe, S. The problem of synergism and antagonism of combined drugs. ArzneimittelForschung 3, 285-290 (1953).

6. BLISS, C.I. THE TOXICITY OF POISONS APPLIED JOINTLY1. Annals of Applied Biology 26, 585-615 (1939).

7. Yadav, B., Wennerberg, K., Aittokallio, T. \& Tang, J. Searching for Drug Synergy in Complex Dose-Response Landscapes Using an Interaction Potency Model. Computational and structural biotechnology journal 13, 504-513 (2015).

8. Malyutina, A. et al. Drug combination sensitivity scoring facilitates the discovery of synergistic and efficacious drug combinations in cancer. PLoS computational biology 15 , e1006752 (2019).

9. Chou, T.C. Drug combination studies and their synergy quantification using the ChouTalalay method. Cancer research 70, 440-446 (2010).

10. Twarog, N.R., Stewart, E., Hammill, C.V. \& Shelat, A.A. BRAID: A Unifying Paradigm for the Analysis of Combined Drug Action. Scientific reports 6, 25523 (2016).

11. Di Veroli, G.Y. et al. Combenefit: an interactive platform for the analysis and visualization of drug combinations. Bioinformatics (Oxford, England) 32, 2866-2868 (2016).

12. lanevski, A., He, L., Aittokallio, T. \& Tang, J. SynergyFinder: a web application for analyzing drug combination dose-response matrix data. Bioinformatics (Oxford, England) 33, 2413-2415 (2017).

13. Wooten, D.J. \& Albert, R. synergy - A Python library for calculating, analyzing, and visualizing drug combination synergy. Bioinformatics (Oxford, England) (2020).

14. lanevski, A., Giri, A.K. \& Aittokallio, T. SynergyFinder 2.0: visual analytics of multi-drug combination synergies. Nucleic acids research 48, W488-w493 (2020).

15. Cokol-Cakmak, M., Cetiner, S., Erdem, N., Bakan, F. \& Cokol, M. Guided screen for synergistic three-drug combinations. PloS one 15, e0235929 (2020).

16. Meyer, C.T., Wooten, D.J., Lopez, C.F. \& Quaranta, V. Charting the Fragmented Landscape of Drug Synergy. Trends in pharmacological sciences 41, 266-280 (2020).

17. Vlot, A.H.C., Aniceto, N., Menden, M.P., Ulrich-Merzenich, G. \& Bender, A. Applying synergy metrics to combination screening data: agreements, disagreements and pitfalls. Drug discovery today 24, 2286-2298 (2019).

18. Bazgir, O. et al. Representation of features as images with neighborhood dependencies for compatibility with convolutional neural networks. Nature communications 11, 4391 (2020).

19. Altman, D.G. \& Bland, J.M. How to obtain the P value from a confidence interval. BMJ (Clinical research ed.) 343, d2304 (2011). 
20. Chambers, J. et al. UniChem: extension of InChl-based compound mapping to salt, connectivity and stereochemistry layers. Journal of cheminformatics 6, 43 (2014).

21. Tang, J. et al. Drug Target Commons: A Community Effort to Build a Consensus Knowledge Base for Drug-Target Interactions. Cell chemical biology 25, 224-229.e222 (2018).

22. Bairoch, A. The Cellosaurus, a Cell-Line Knowledge Resource. Journal of biomolecular techniques : JBT 29, 25-38 (2018).

23. Ansbro, M.R. et al. Modulation of Triple Artemisinin-Based Combination Therapy Pharmacodynamics by Plasmodium falciparum Genotype. ACS pharmacology \& translational science 3, 1144-1157 (2020).

24. Zagidullin, B. et al. DrugComb: an integrative cancer drug combination data portal. Nucleic acids research 47, W43-w51 (2019).

25. O'Neil, J. et al. An Unbiased Oncology Compound Screen to Identify Novel Combination Strategies. Molecular cancer therapeutics 15, 1155-1162 (2016).

26. Preuer, K. et al. DeepSynergy: predicting anti-cancer drug synergy with Deep Learning. Bioinformatics (Oxford, England) 34, 1538-1546 (2018).

27. Menden, M.P. et al. Community assessment to advance computational prediction of cancer drug combinations in a pharmacogenomic screen. Nature communications 10, 2674 (2019).

28. Li, H., Li, T., Quang, D. \& Guan, Y. Network Propagation Predicts Drug Synergy in Cancers. Cancer research 78, 5446-5457 (2018).

29. Zheng, S. et al. DrugComb update: a more comprehensive drug sensitivity data repository and analysis portal. BioRxiv, 2021.2003.2025.436916 (2021).

30. Liu, $\mathrm{H}$. et al. DrugCombDB: a comprehensive database of drug combinations toward the discovery of combinatorial therapy. Nucleic acids research 48, D871-D881 (2020).

31. Douglass, E.F. et al. A Community Challenge for Pancancer Drug Mechanism of Action Inference from Perturbational Profile Data. BioRxiv, 2020.2012.2021.423514 (2020).

32. Zhang, B. et al. The tumor therapy landscape of synthetic lethality. Nature communications 12, 1275 (2021).

33. Shah, K., Ahmed, M. \& Kazi, J.U. The Aurora kinase/ $\beta$-catenin axis contributes to dexamethasone resistance in leukemia. NPJ precision oncology 5, 13 (2021).

34. Jones, P.A., Ohtani, H., Chakravarthy, A. \& De Carvalho, D.D. Epigenetic therapy in immune-oncology. Nature reviews. Cancer 19, 151-161 (2019).

35. Fernandes Neto, J.M. et al. Multiple low dose therapy as an effective strategy to treat EGFR inhibitor-resistant NSCLC tumours. Nature communications 11, 3157 (2020).

36. Ozkan-Dagliyan, I. et al. Low-Dose Vertical Inhibition of the RAF-MEK-ERK Cascade Causes Apoptotic Death of KRAS Mutant Cancers. Cell reports 31, 107764 (2020).

37. Caumanns, J.J. et al. Low-dose triple drug combination targeting the PI3K/AKT/mTOR pathway and the MAPK pathway is an effective approach in ovarian clear cell carcinoma. Cancer letters 461, 102-111 (2019).

38. Van Cutsem, E. et al. Binimetinib, Encorafenib, and Cetuximab Triplet Therapy for Patients With BRAF V600E-Mutant Metastatic Colorectal Cancer: Safety Lead-In Results From the Phase III BEACON Colorectal Cancer Study. Journal of clinical oncology : official journal of the American Society of Clinical Oncology 37, 1460-1469 (2019).

39. Greco W, U.H.-D., Pöch G, Sühnel J, Kundi M, Bödeker W CONSENSUS ON CONCEPTS AND TERMINOLOGY FOR COMBINEDACTION ASSESSMENT: THE SAARISELKA AGREEMENT. Arch. Complex Environ. Stud. 4, 65-69 (1992).

40. Tang, J., Wennerberg, K. \& Aittokallio, T. What is synergy? The Saariselkä agreement revisited. Frontiers in pharmacology 6, 181 (2015).

41. Wood, K., Nishida, S., Sontag, E.D. \& Cluzel, P. Mechanism-independent method for predicting response to multidrug combinations in bacteria. Proceedings of the National Academy of Sciences of the United States of America 109, 12254-12259 (2012).

42. Adam, G. et al. Machine learning approaches to drug response prediction: challenges and recent progress. NPJ precision oncology 4, 19 (2020).

43. Kim, Y. et al. Anticancer drug synergy prediction in understudied tissues using transfer learning. Journal of the American Medical Informatics Association : JAMIA 28, 42-51 (2021). 


\section{Supplementary files}

Supplementary Figure 1. Two-dimensional surface plots of synergy scores. A) HSA, B) BLISS and C) LOEWE, for a synergistic three-drug combination Pyronaridine Tetraphosphate-ArtesunateDarunavir Ethanolate (left panel) compared to an antagonistic drug combination (Pyronaridine Tetraphosphate-Piperaquine-Lopinavir, right panel).

Supplementary Figure 2. Synergy-sensitivity plots for HSA, BLISS and LOEWE scores in relation to the combination sensitivity score for the drug pairs tested in the A2058 cell line. 
bioRxiv preprint doi: https://doi.org/10.1101/2021.06.01.446564; this version posted June 3, 2021. The copyright holder for this preprint (which

was not certified by peer review) is the author/funder, who has granted bioRxiv a license to display the preprint in perpetuity. It is made available under aCC-BY-NC-ND 4.0 International license.

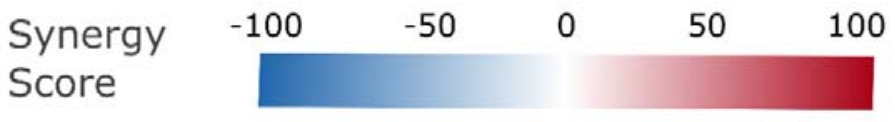

\section{HSA}
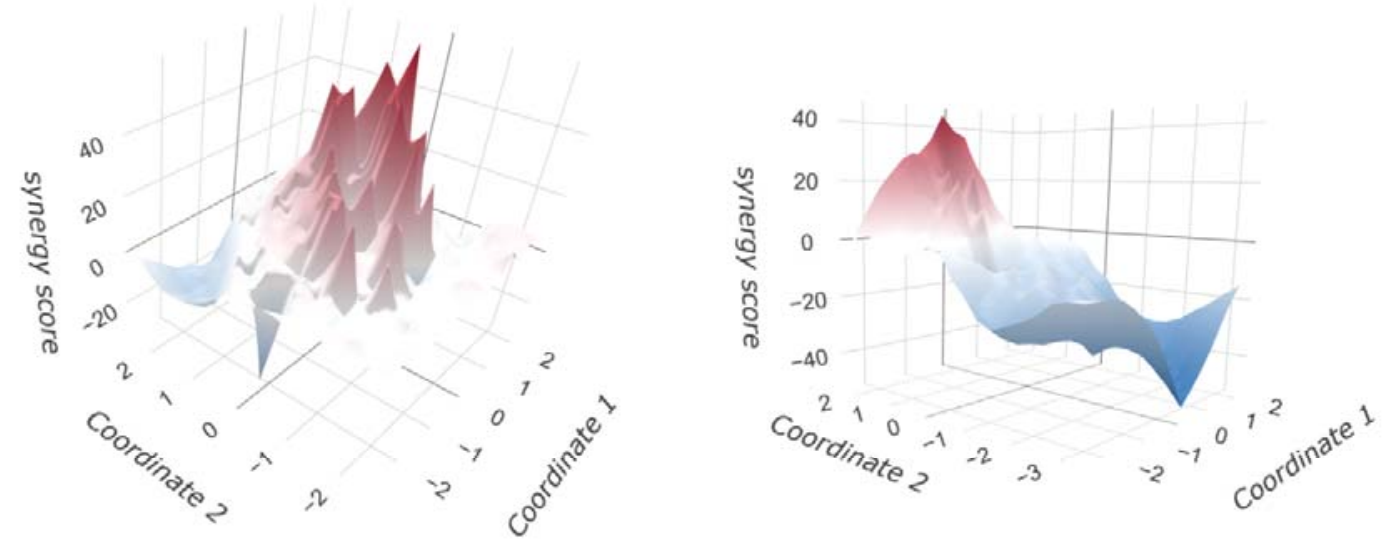

\section{Bliss}
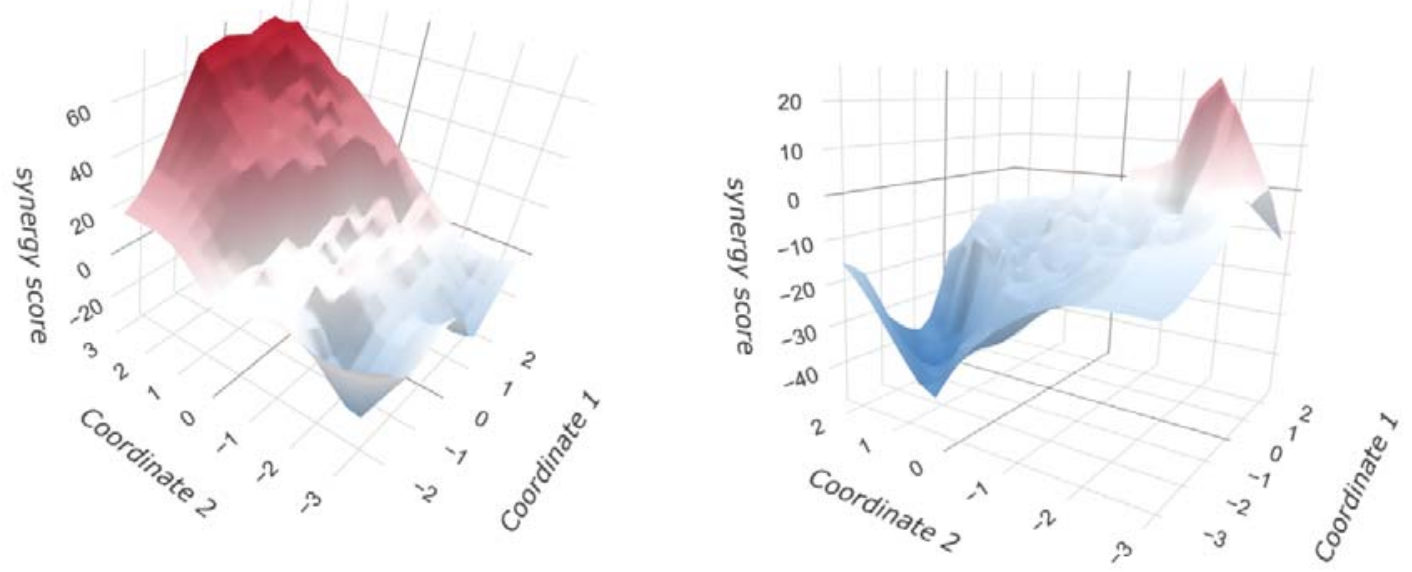

\section{Loewe}
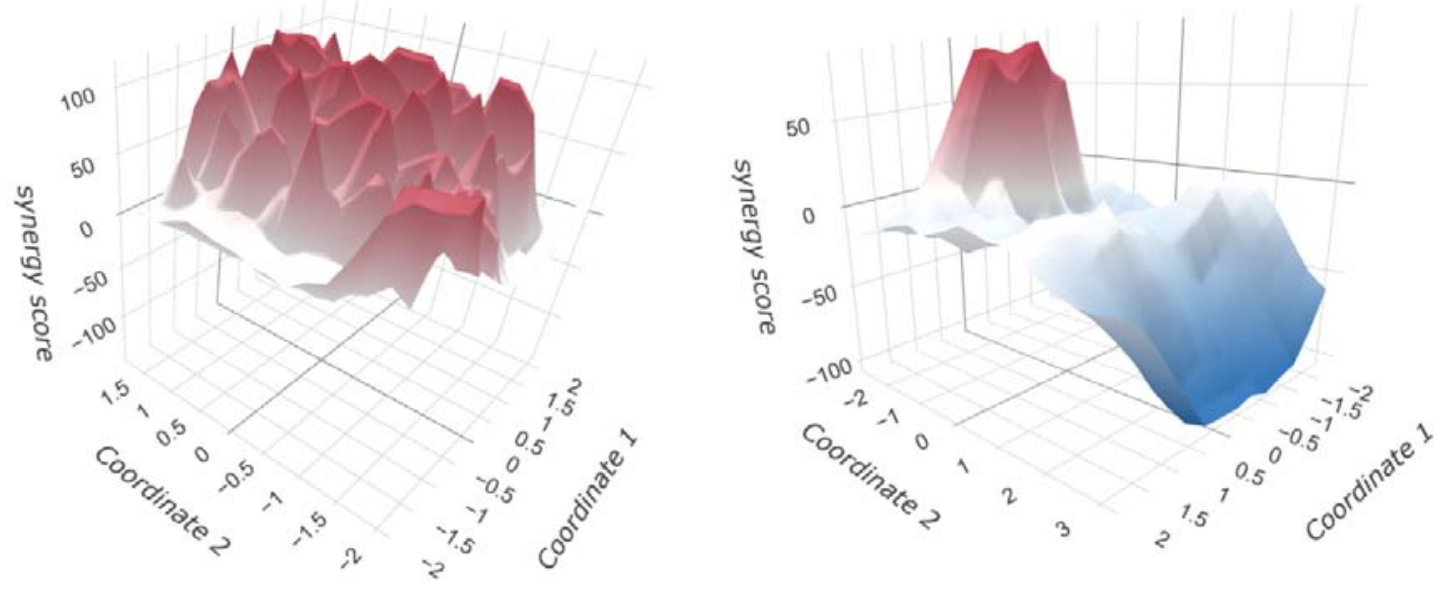
bioRxiv preprint doi: https://doi.org/10.1101/2021.06.01.446564; this version posted June 3, 2021. The copyright holder for this preprint (which was not certified by peer review) is the author/funder, who has granted bioRxiv a license to display the preprint in perpetuity. It is made available under aCC-BY-NC-ND 4.0 International license.
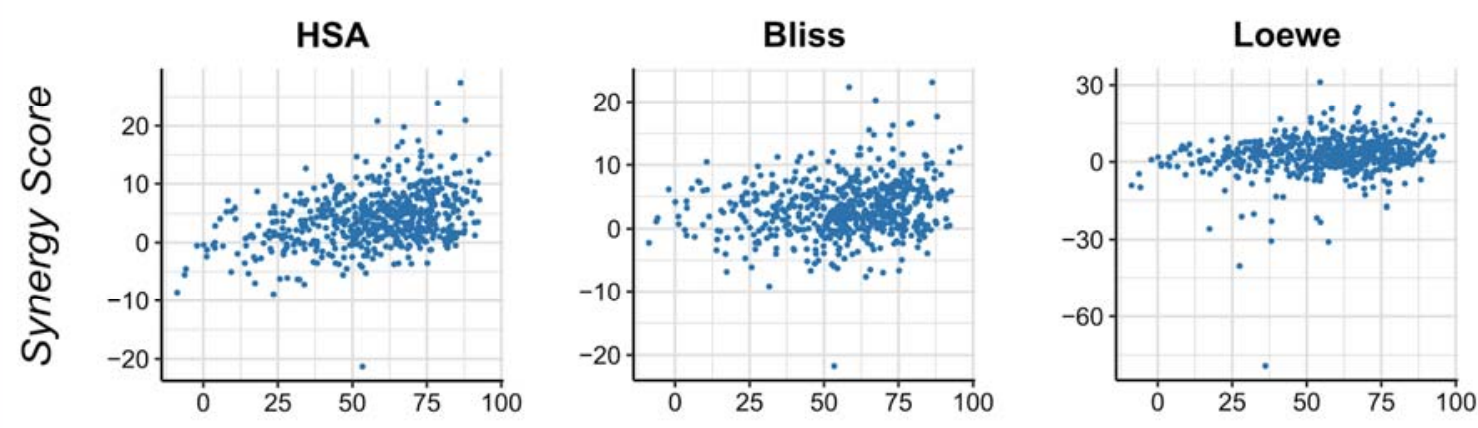

Combination Sensitivity Score 